\title{
Reducing Radiation Dose by Using Pulse X-Ray Apparatus
}

\author{
A. A. Komarskiy1,2, A. S. Chepusov'1,2, V. L. Kuznetsov¹, S. R. Korzhenevskiy'1, S. P. Niculin1,2, \\ S. O. Cholakh ${ }^{2}$ \\ ${ }^{1}$ Pulsed Radiation Sources Laboratory, The Institute of Electrophysics of the Ural Division of the Russian \\ Academy of Science, Ekaterinburg, Russian Federation \\ ${ }^{2}$ Institute of Physics and Technology, Ural Federal University (Named after First President of Russia B.N. Yeltsin), \\ Ekaterinburg, Russian Federation \\ Email: aakomarskiy@gmail.com
}

Received December 2013

\begin{abstract}
Pulse X-ray diagnostics is capable of reducing the radiation exposure considerably. As for pulse $\mathrm{X}$-ray diagnostic machines, which form pulses with the duration of $0.1 \mu \mathrm{s}$, using them one can get outstanding results in this area. This fact can be explained by the long period of luminophor persistence in intensifying $X$-ray luminescent screens. In this paper we present experimental data, comparing radiation doses, measured at pulse $\mathrm{X}$-ray apparatus and apparatus of constant radiation.
\end{abstract}

Keywords

X-Radiation; Pulse X-Ray Tube; Pulse X-Ray Diagnostic; Ionizing Radiation

\section{Introduction}

Medical radiation makes up a major portion of the radiation dose for population of the Earth. As for the Russian Federation this type of radiation takes the second place after natural sources of ionizing radiation in the total radiation doze. $98 \%$ of the radiation is taken by diagnostic and prophylactic X-ray examinations.

Pulse X-ray diagnostics is capable of reducing the radiation exposure considerably. Generating X-ray radiation as a sequence of short X-ray flashes instead of continuous radiation is a distinguishing feature of this method. The pulse X-ray tube (pulse duration 5 - 20 ms, frequency 2 - 12 pulses per second), which is used during endoscopic diagnostic and therapeutic interventions of bile ducts, was described in paper [1]. The shorter the pulse and the less the frequency - the less the radiation exposure of the patient and the personnel, but the quality of the images reduces accordingly.

As for pulse X-ray diagnostic machines, which form pulses with the durations of $0.1 \mu \mathrm{s}$, the principle of reducing the absorbed dose is completely different.

\section{Experiment}

In medical diagnostics intensifying X-ray luminescent screens are obligatory. Since X-ray film, which most 
commonly used to absorbed radiation has coefficient of X-ray radiation 0.01 , as for intensifying screens Gd2O2S:Tb the coefficient is 0.29 [2]. Luminophors, which are used in the intensifying screens, have a certain period of persistence [3].

To define the duration of persistence precisely timing data and fluorescence intensity of luminophors, used in diagnostics, Kodak lanex, Renex EU-G3, Renex EU-G300, Renex EU-I4, were measured. Measuring was performed at a pulsed cathodoluminescence spectrometer. In Figure 1, you can see an oscillogram, picturing the process of luminophor persistence Renex EU-G3, typical for all the group of intensifying screens under investigation. The persistence duration of level 2.7 is $500 \mu$ s when pulse duration is $0.1 \mu \mathrm{s}$.

The final results as persistence duration of the intensifying screens of level 0.1 are given in Figure 2.

Measuring amplitude-time characteristics of persistence show, that all luminophors under investigation are characterized by the persistence at level of a millisecond, which is by an order of magnitude greater than radiation pulse duration of a nanosecond $\mathrm{X}$-ray unit, as shown in Figure 3.

$\mathrm{X}$-ray quanta irradiate luminophor the $\mathrm{X}$-ray photo detector luminophor with the intensity $\mathrm{I}_{\mathrm{r}}$, and the luminophor radiation intensity is

$$
I_{l}(t)=I_{0} \cdot e^{\frac{-\tau}{T}}
$$

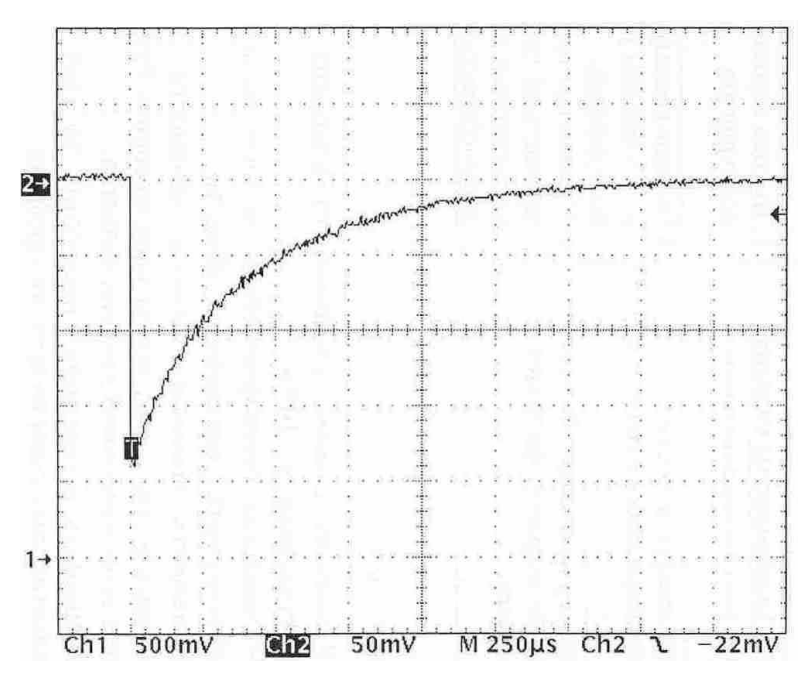

Figure 1. The persistence X-ray intensifying screen Renex EU-G3.

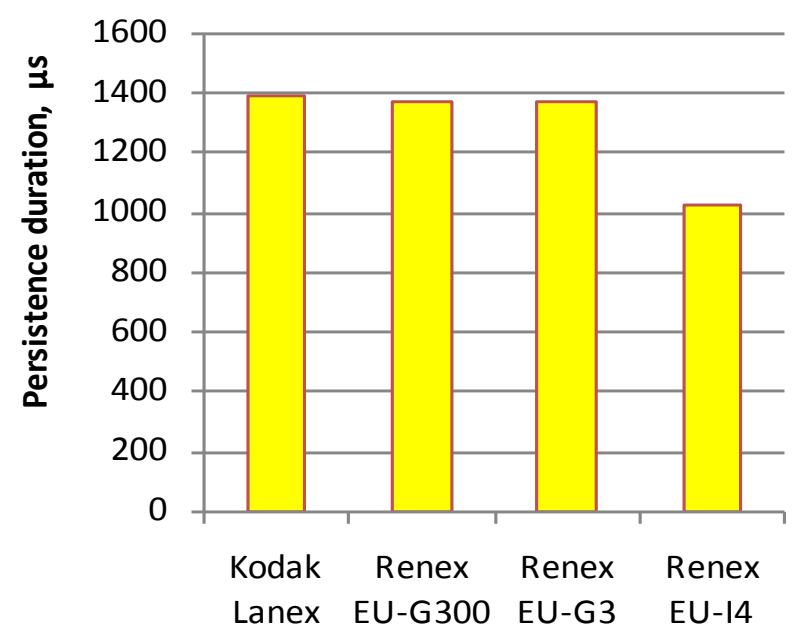

Figure 2. Persistence duration of the intensifying screens of level 0,1. 


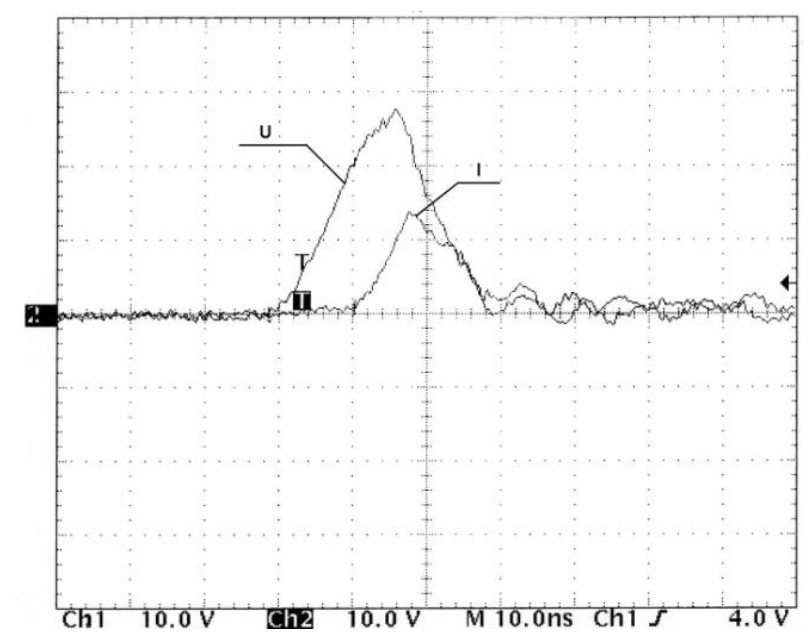

Figure 3. An oscillogram of voltage and current of the X-ray tube RIA1-120. Voltage amplitude-110 kV, current amplitude $-450 \mathrm{~A}$, scale $10 \mathrm{~ns} /$ point, repetition rate $850 \mathrm{~Hz}$.

where $I_{l}(t)$ is the radiation intensity at the time $t, \tau$ is the average time during which the excited state of luminophor atoms or molecules persists, $T$ is period, $I_{0}$ is radiation intensity at the time when excitation of luminescence stops

$$
I_{0} \approx(0.2-0.01) \cdot I_{r}
$$

when using a constant X-radiation source, luminophor generates light radiation with the intensity $I_{0}$ and a persistence fall after X-ray exposure in $e$ times within $(1-1.5) * 10^{-3}$ (s) during the whole time of exposure from hundreds of milliseconds and higher. At pulse radiation after X-ray pulse exposure luminophor persistence corresponds to $e$ times during the same time, but this process repeats after each pulse. In the result of X-ray film accumulating light sum of the $\mathrm{X}$-radiation, converted by luminophor, after $\mathrm{X}$-ray pulse stops, the great difference in duration of the X-ray pulse and the luminophor persistence fall makes it possible to reduce the exposure dose. At the Institute of Electrophysics of the Ural Division of the Russian Academy of Science we invented the pulse X-ray diagnostic portable apparatus 01 (XDPA 01) [4], which was based on the described effect. Then we upgraded the X-ray apparatus and created the pulse X-ray diagnostic portable apparatus Yasen-01 [5] [6].

We performed dosimetric tests, comparing the pulse X-ray diagnostic portable apparatus Yasen-01 (maximum voltage is $110 \mathrm{kV}$, the tube's current is $150 \mathrm{~A}$, pulse duration is $30 \mathrm{~ns}$, repetition rate is $5 \mathrm{kHz}$ ) with the X-ray diagnostic apparatus Siemens Axiom Iconos R200 (maximum voltage is $125 \mathrm{kV}$ ), using a direct current tube.

Measuring dosimetric parameters was carried out with the help of the dosimeter PTW UNIDOS 10001 with $30 \mathrm{~cm}^{3}$ cylindrical ionization chamber of 23361 kinds. Several standard modes of the tested machines for medical diagnostics of different body organs were chosen. The main criterion for the comparison was the diagnostic quality of the X-ray photographs, as shown in Figure 4.

Water phantom was used behind the ionization chamber to imitate a human body (as a scattered radiation source). Phantom thickness was $20 \mathrm{~cm}$, focal distance to the ionization chamber was $80 \mathrm{~cm}$. The results of investigations are shown in Table 1.

Then we performed tests, comparing the pulse X-ray apparatus Yasen-01 with the X-ray portable diagnostic apparatus Definium AMX 700 (maximum voltage is $105 \mathrm{kV}$ ) and with stationary X-ray complex Evolution HV which was created by STEPHANIX (maximum voltage is $40-150 \mathrm{kV}$ ). We compared the following parameters: effective doses, the quality of the X-ray images and usability.

Calculating the effective doses of patients was made using the results of measuring radiation output of the apparatus with the help of the following measuring devices: the wide-range device for dosimetry of continuous and pulse X-rays and gamma rays DKS-AT1123, the all-mode dosimeter for controlling characteristics of X-ray apparatus-Unfors Xi RF\&MAM detector. In Table 2, effective doses of patient irradiation are presented.

Three proficient roentgenologists carried out independent estimation of the X-ray images' quality and the apparatus' usability. 


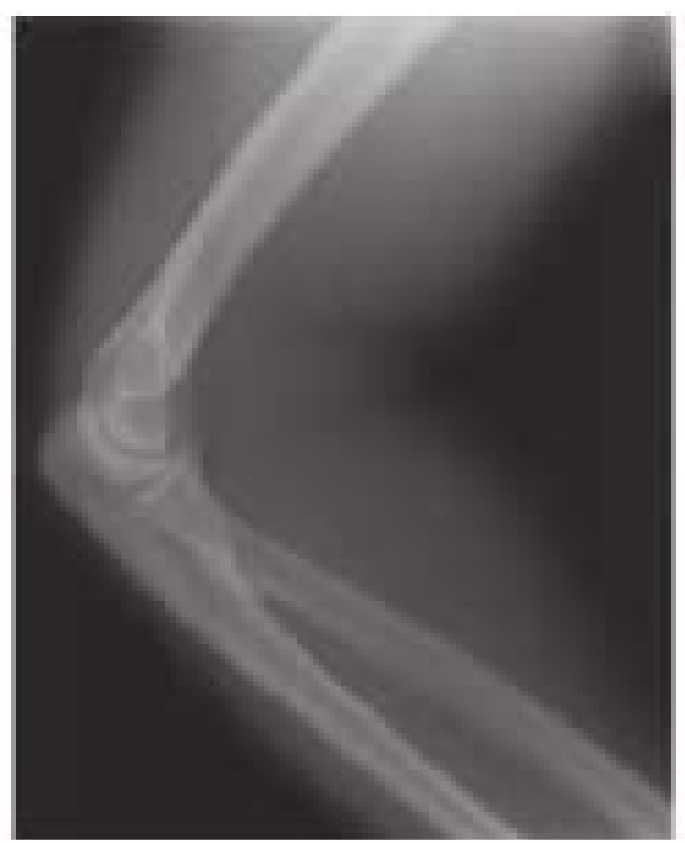

(a)

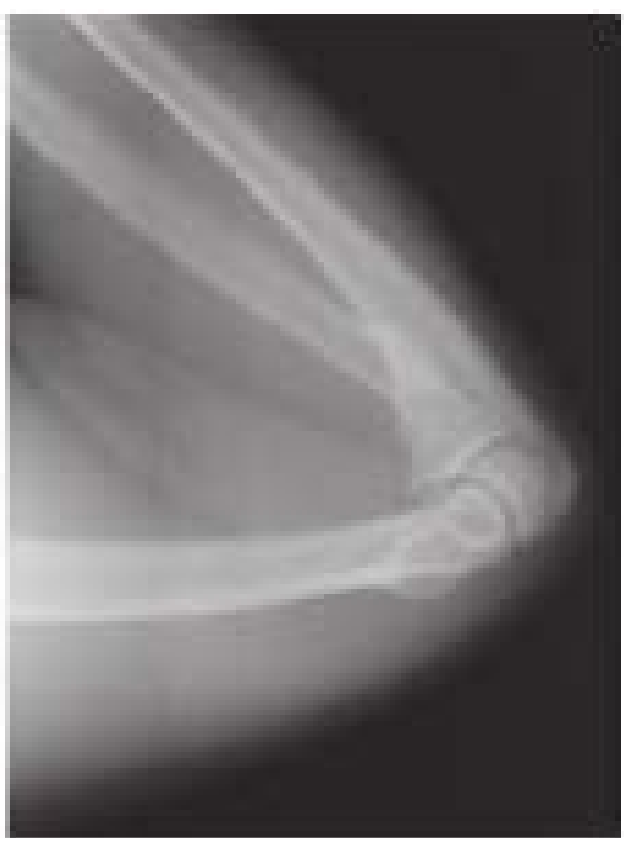

(b)

Figure 3. Elbow arthrogram using by a) X-ray diagnostic apparatus with direct current tube; b) pulse X-ray diagnostic portable apparatus Yasen-01.

Table 1. Test results of Siemens Axiom Iconos R200 and Yasen-01.

\begin{tabular}{ccccccc}
\hline \multirow{2}{*}{ Object, projection } & \multicolumn{3}{c}{ Siemens Axiom Iconos R200 } & \multicolumn{3}{c}{ Yasen-01 } \\
\cline { 2 - 7 } & Voltage, $\mathrm{kV}$ & Exposition, mAs & Dose, $\mu \mathrm{Gy}$ & Voltage, $\mathrm{kV}$ & Exposition, $\mathrm{mAs}$ & Dose, $\mu \mathrm{Gy}$ \\
\hline Lungs, direct & 57 & 3.6 & 200 & 110 & 1.4 & 70 \\
Skull, side & 60 & 8 & 505 & 110 & 3.7 & 185 \\
Thigh, direct & 55 & 8 & 410 & 110 & 3.4 & 170 \\
Knee, direct & 47 & 6.3 & 215 & 100 & 1.8 & 86 \\
Ankle, direct & 48 & 4.5 & 160 & 100 & 1.0 & 48 \\
\hline
\end{tabular}

Table 2. Effective doses of patient irradiation.

\begin{tabular}{ccc}
\hline Name & Projection & Effective dose, mSv \\
\hline Yasen-01 & Chest (direct) & 0.027 \\
Definium AMX 700 & Chest (direct) & 0.570 \\
Evolution HV & Chest (direct) & 0.721 \\
\hline
\end{tabular}

\section{Discussion}

As follows from the Table 1, the radiation dose of Yasen-01 is 2.5 - 3 times lower than the one of Siemens Axiom Iconos R200 and 20 - 25 times lower compared with Definium AMX 700, Evolution HV. The fact is that with the help of the pulse X-ray we can decrease the dose more significantly can be explained by the higher value of anode current (Yasen-01 - $150 \mathrm{~A}$ ), since luminophor radiation intensity $\mathrm{I}_{1}$ is proportional to $\mathrm{X}$-ray quantum intensity $\mathrm{I}_{\mathrm{r}}$ hence it proportional to the $\mathrm{X}$-ray tube current $\mathrm{I}_{\mathrm{e}}$. Moreover, decreasing dose is explained by pulse duration: the Yasen-01 apparatus generates X-ray pulses of shorter duration (30 ns). Therefore the Yasen-01 apparatus has a more homogeneous radiation spectrum and greater effective energy $(42.5 \mathrm{keV})$, i.e. it 
creates harder radiation. The X-ray tube's performance in good conditions in terms of maximum acceptable heat load of the anode should be considered as one of the machines' advantages. At present the operating mode of constant radiation machines is limited to $0.3-0.01 \mathrm{~A}$. This limitation is subject to maximum acceptable heat load of the anode, since exceeding this value causes anode destruction. However the pulse tube current reaches the peak value of $150 \mathrm{~A}$, which increases luminescence intensity $I_{l}$ by dozens of times. For example, if repetition rate is $f=1 \mathrm{kHz}$ and pulse durations is $\tau=10^{-8} \mathrm{~s}$, duty factor

$$
Q=\frac{1}{f \cdot \tau}
$$

equals $Q=10^{5}$, and the tube average current $<I>$ (when current pulse is $I_{e}=100 \mathrm{~A}$ ) is inversely proportional to pulse duty factor,

$$
<I>=\frac{I_{e}}{Q}
$$

and equals just $<I>=1 \times 10^{-3} \mathrm{~A}$.

The chest X-Ray direct projection images, which were obtained at the pulse X-ray diagnostic portable apparatus Yasen-01, are the same as to the quality of the images, which were obtained by the X-ray portable diagnostic apparatus Definium AMX 700 and are insignificantly inferior compared with the images obtained by the stationary X-ray complex Evolution HV which was created by STEPHANIX.

Roentgenologist point out that thanks to the compact size and low weight (about $45 \mathrm{~kg}$ ) the Yasen-01 apparatus can be used in confined space and is easily transported.

\section{Conclusions}

In conclusion, the conducted investigation demonstrates that X-ray radiation dose is decreased by several times thanks to using pulsed nanosecond X-ray machines as opposed to using relative conventional X-ray units. The dose of radiation was reduced so significantly thanks to the fact that persistence duration of $\mathrm{X}$-ray radiation converters (luminophor) is several times higher than X-ray pulse duration.

Further decreasing radiation dose is possible owing to increasing current pulse amplitude $I_{e}$, shortening pulse duration $\tau$ and increasing duty factor $Q$.

The quality of the X-Ray images which were obtained at the pulse X-ray diagnostic portable apparatus Yasen-01 is the same as at constant $\mathrm{X}$-radiation apparatus. Thanks to its small size and weight Yasen-01 apparatus can be used in confined space and easily transported in narrow corridors and elevators.

\section{References}

[1] Doroshko, M. V. (1998) New Capabilities in Reducing of Radiation Dose лучевой нагрузки. News of Radiodiagnostics, 3, 13-15.

[2] Blinov, N.N., Bikov, R.E. and Kozlovskiy, E.B. (1989) Engineering Tools of Medical Introscopy. Leonov B.I., Moskow.

[3] Kazgikin, O.N., Markovskiy, L.Y., Mironov, I.A., Pskerman, F.M. and Petoshina, L.N. (1975) Inorganic Phosphor. Leningrad.

[4] Filatov, A.L., Korzhenevskiy, S.R., Kuznetsov, V.L., Ananin, M.V. and Motovilov, V.A. (2004) The Nanosecond PP X-ray Apparatus. Procedings of the 15th International Conference on High-Power Particle Beams, Saint-Petersburg, 1-7 April 2004, 552-554.

[5] Filatov, A.L., Bastrikov, V.L., Korzhenevskiy, S.R., Kuznetsov, V.L. and Ponikaravskih, A.E. (2007) Universal Portable X-Ray Apparatus. Russian Federation Patent No. 2005503167.

[6] Mozharova I.E. and Kuznetsov V.L. (2011) The Pulse X-Ray Diagnostic Portable Apparatus “Yasen 01”. Medical business, 9, 84-85. 